\title{
OX-1 metal-organic framework nanosheets as robust hosts for highly active catalytic palladium species
}

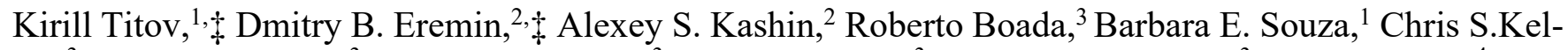 \\ ley, ${ }^{3}$ Mark D. Frogley, ${ }^{3}$ Gianfelice Cinque, ${ }^{3}$ Diego Gianolio, ${ }^{3}$ Giannantonio Cibin, ${ }^{3}$ Svemir Rudić, ${ }^{4}$ Val- \\ entine P. Ananikov, ${ }^{2, *}$ and Jin-Chong Tan ${ }^{1, *}$ \\ ${ }^{1}$ Multifunctional Materials \& Composites (MMC) Laboratory, Department of Engineering Science, University of Oxford, \\ Parks Road, Oxford OX1 3PJ, United Kingdom \\ ${ }^{2}$ Zelinsky Institute of Organic Chemistry, Russian Academy of Sciences, Leninsky Pr. 47, Moscow 119991, Russia \\ ${ }^{3}$ Diamond Light Source, Harwell Campus, Chilton, Didcot, OX11 0DE, United Kingdom \\ ${ }^{4}$ ISIS Facility, STFC, Rutherford Appleton Laboratory, Chilton, Didcot OX11 0QX, United Kingdom \\ \$ These authors contributed equally to this work \\ E-mail: *V.P.A.: val@ioc.ac.ru; *J.C.T.: jin-chong.tan@eng.ox.ac.uk \\ KEYWORDS: nanosheets, metal-organic framework, palladium, catalysis, inelastic scattering.
}

\begin{abstract}
A catalytic system based on OX-1 metal-organic framework nanosheets is reported, incorporating catalytically active palladium (Pd) species. The Pd@OX-1 guest@host system is rapidly synthesised via a one-step single-pot supramolecular assembly, with the possibility of controlling the Pd loading. The structures of the resulting framework and of the active Pd species before and after catalytic reactions are studied in detail using a wide variety of techniques including synchrotron radiation infrared spectroscopy, inelastic neutron scattering and X-ray absorption spectroscopy. Crystals of the resulting Pd@OX-1 composite material contain predominantly atomic and small cluster Pd species, which selectively reside on benzene rings of the benzenedicarboxylate (BDC) linkers. The composites are shown to efficiently catalyse the Suzuki coupling and Heck arylation reactions under a variety of conditions. $\mathrm{Pd} @ \mathrm{OX}-1$ further shows potential to be recycled for at least five cycles of each reaction as well as an ability to recapture active Pd species during both catalytic reactions.
\end{abstract}

\section{INTRODUCTION}

The vast chemical and physical versatility of metal-organic framework (MOF) materials opens the door to a range of potential technological applications: adsorption, catalysis, charge transport and luminescence, photonics and chemical sensing, and delivery of functional guest molecules. ${ }^{1,2}$ There has been a mounting interest in the exploration of MOF compounds or MOF-based composites to afford heterogenous catalysis, summarized by recent reviews in this topic area.-6 Interesting findings include the demonstration that the activity of MOF catalysts containing coordinatively unsaturated sites, such as UiO-66, can be significantly enhanced by functionalised linkers. ${ }^{7}$ A related study shows that catalytic activity of MIL-100(Fe) can be substantially enhanced and tuned by introducing defects in the structure via acid pretreatment. ${ }^{8}$ In the study of MOF-based composites as catalysts, an emerging avenue involves the immobilisation of fine-scale metal nanoparticles confined in the high-surface-area MOF hosts, ${ }^{9}$ yielding bespoke composites exhibiting a good combination of chemical and mechanical properties. ${ }^{10}$

Metal-organic nanosheets ${ }^{11,} 12$ and hybrid nanoplate structures ${ }^{13}$ present a two-dimensional (2-D) platform not only for engineering thin-film devices ${ }^{14,15}$ but also showing promise for photocatalytic applications. ${ }^{16}$ Recently, we have discovered a porous $\mathrm{Zn}$-based nanosheet structure designated as $\mathrm{OX}-1$ for "Oxford University-1 material" $\left[\left(\mathrm{Et}_{3} \mathrm{NH}\right)_{2} \mathrm{Zn}_{3} \mathrm{BDC}_{4} ; \mathrm{Et}_{3} \mathrm{~N}=\right.$ triethylamine, $\mathrm{BDC}=1,4$-benzenedicarboxylate], which may serve as an efficient host structure to confine (guest) luminescent metal complexes. ${ }^{17}$ It demonstrated the interesting concept of guest@MOF (host) strategy, ${ }^{18}$ enabled by a rapid one-pot supramolecular assembly approach under ambient conditions. It is envisaged that this guest@MOF strategy may be extended to achieve the in situ encapsulation of fine-scale metal nanoparticles as catalytically active species.

The catalytic formation of $\mathrm{C}-\mathrm{C}$ bonds is considered to be one of the key transformations in organic synthesis. ${ }^{19}$ The use of transition metals as catalysts for these transformations is a vibrant area of research, especially involving metal nanoparticles. ${ }^{20-25}$ The precise processes involved in nanoparticle catalysis are a matter of ongoing investigations, ${ }^{26}$ while the ultimate aim is the rational design of efficient, selective and stable catalysts, with an awareness of the dynamic evolution of the active species during catalysis. ${ }^{26-30}$ 

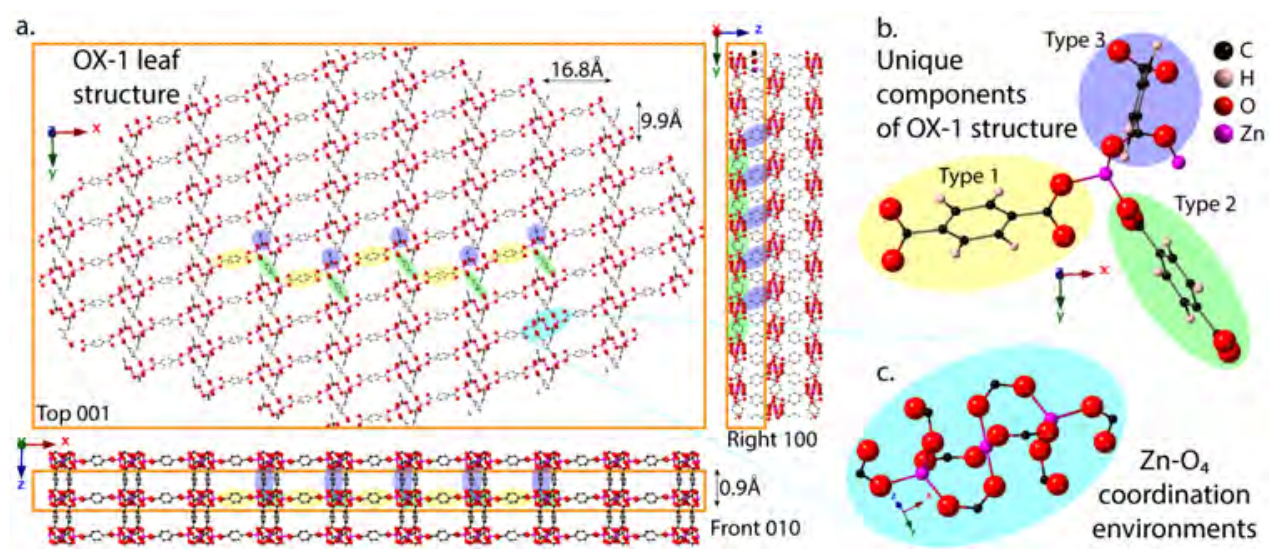

Figure 1. Crystal structure of OX-1 nanosheets exhibiting a leaf-shaped morphology: (a) views of a selected 2-D layer (bound by the orange box) making up the 3-D sheet structure; (b) details of the unique components of the structure -2 unique $\mathrm{Zn}$ centres and 3 possible orientations of BDC linker; (c) detail of the $\mathrm{Zn}$ coordination environments. Coloured ovals highlight the positions of the different types of structural motifs in the crystal structure.

MOF materials possess unique properties, such as tunable porosity and the ability to fine tune the structure of the active sites and their environments, ${ }^{31}$ which can be utilised in achieving the above aims. MOF catalytic activity can be achieved via three main routes: activity at the inorganic nodes and the organic, or pseudo-organic, linkers of the framework itself; encapsulation of active species into the framework; and post-synthetic modification of the framework. ${ }^{31-35}$ Some notable examples of catalytic MOFs for C-C bond formation so far have been achieved through encapsulation of active palladium (Pd) species into the MIL family of MOFs, ${ }^{34,} 36-41$ as well as $\mathrm{UiO}-66^{42}$, UiO-6743, MIL-53(Al)-NH${ }_{2}^{44}$, ScBTC NMOF ${ }^{45}$, $\mathrm{Cu}_{2}(\mathrm{BDC})_{2} \mathrm{DABCO}{ }^{46}$, and MOF- $5^{47}$. These materials have been shown to catalyse the Suzuki reaction for a wide range of substrates, but few demonstrate catalysis of the Heck reaction ${ }^{40}$, 44. However, these MOFs are difficult and resource-intensive to synthesise, often requiring prolonged heating in autoclave enclosures, and in all cases requiring a long secondary processing step to incorporate the active $\mathrm{Pd}$ phase into the framework. The present work adddresses these difficulties without compromising the high activity at low loadings of incorporated Pd species.

In this study we report a new catalytic system based on OX-1 nanosheets, incorporating catalytically active Pd species. The Pd@OX-1 guest@host system is rapidly synthesised via a one-step single-pot supramolecular assembly, with the possibility of controlling the Pd loading. The structures of the resulting framework and of the active Pd species before and after catalytic reaction are studied in detail using a wide variety of techniques including synchrotron radiation infrared (SRIR) spectroscopy, inelastic neutron scattering (INS) and X-ray absorption spectroscopy (XAS). Pd@OX-1 is shown to be a highly efficient catalyst of the Suzuki coupling and Heck arylation reactions, with the possibility of recycling the material for at least five cycles of each reaction. Furthermore, OX-1 is shown to possess active sites for Pd attachment, which are not deactivated in the process of the Heck reaction and even survive a complete transformation of crystal structure during the Suzuki reaction.

\section{EXPERIMENTAL SECTION}

\section{Materials synthesis}

We synthesised a series of OX-1 materials with varying loadings of palladium (Pd), whose zero valency form, $\operatorname{Pd}(0)$, is an active catalyst for a variety of important organic synthesis reactions. Palladium is embedded in the material during OX-1 synthesis and does not affect the processing time. To incorporate $0.1-1 \mathrm{wt} . \%$ of Pd into OX-1, 5-50 $\mathrm{mg}$ of the precursor $\mathrm{Pd}_{2}(\mathrm{dba})_{3}$ complex [dba = dibenzylideneacetone] was dissolved in $2 \mathrm{~mL}$ of DMF and added to $3 \mathrm{mmol}$ of benzene-1,4-dicarboxylic acid and $6 \mathrm{mmol}$ of $\mathrm{Et}_{3} \mathrm{~N}$ in $3 \mathrm{~mL}$ of DMF. This mixture was then combined with $1.5 \mathrm{mmol}$ of $\mathrm{Zn}\left(\mathrm{NO}_{3}\right)_{2}$ in $2 \mathrm{~mL}$ of $\mathrm{DMF}$, at which stage a gel was observed and OX-1 crystals were formed. The gel was then transferred to a $50 \mathrm{~mL}$ centrifuge tube, with $43 \mathrm{~mL}$ of DMF added in the process. This mixture was sonicated until it lost its pink colour, becoming grey-greenish. It was then centrifuged for $5 \mathrm{~min}$ at $8000 \mathrm{rpm}$, to separate the light grey crystals from the light marsh solution of dba and excess BDC in DMF. The crystals were then washed twice in acetone and dried overnight at $100{ }^{\circ} \mathrm{C}$ (yield $\sim 500 \mathrm{mg}$ ). The colour change from pink to greengrey during the first sonication step is evidence of dissociation of the Pd complex. During this step Pd atoms break free of the $\mathrm{Pd}_{2}(\mathrm{dba})_{3}$ complex and bind onto OX-1. Gas chromatography mass spectrometry (GC-MS) and ${ }^{1} \mathrm{H}$ nuclear magnetic resonance (NMR) spectroscopy measurements were carried out to confirm the presence of free dba in solution, showing that $\mathrm{Pd}$ has been dissociated from the $\mathrm{Pd}_{2}(\mathrm{dba})_{3}$ complex. It is important to note that it was not possible to reproduce the Pd@OX-1 material by dispersing pre-synthesised $\mathrm{OX}-1$ crystals in DMF, adding only $\operatorname{Pd}_{2}(\mathrm{dba})_{3}$ to the mixture and sonicating - in this case the white OX-1 turned black, indicating that large Pd particles formed over an extended period of suspension in DMF. Details of the characterisation methods employed in this study are presented in the Supporting Information (SI).

\section{RESULTS AND DISCUSSION}

\section{Crystal structure and scalability}

OX-1 crystal structure ${ }^{17}$, shown in Figure 1, gives rise to 3D leaf-shaped sheet formation in a one-pot supramolecular synthesis. Single layers of $\mathrm{Zn}$ metal centers and BDC linkers (Figure 1(a)) are stacked with an offset of half pore width and a reflection to create the sheets seen in Figure 2(a-c, e-f). This structure is built up using just five unique 

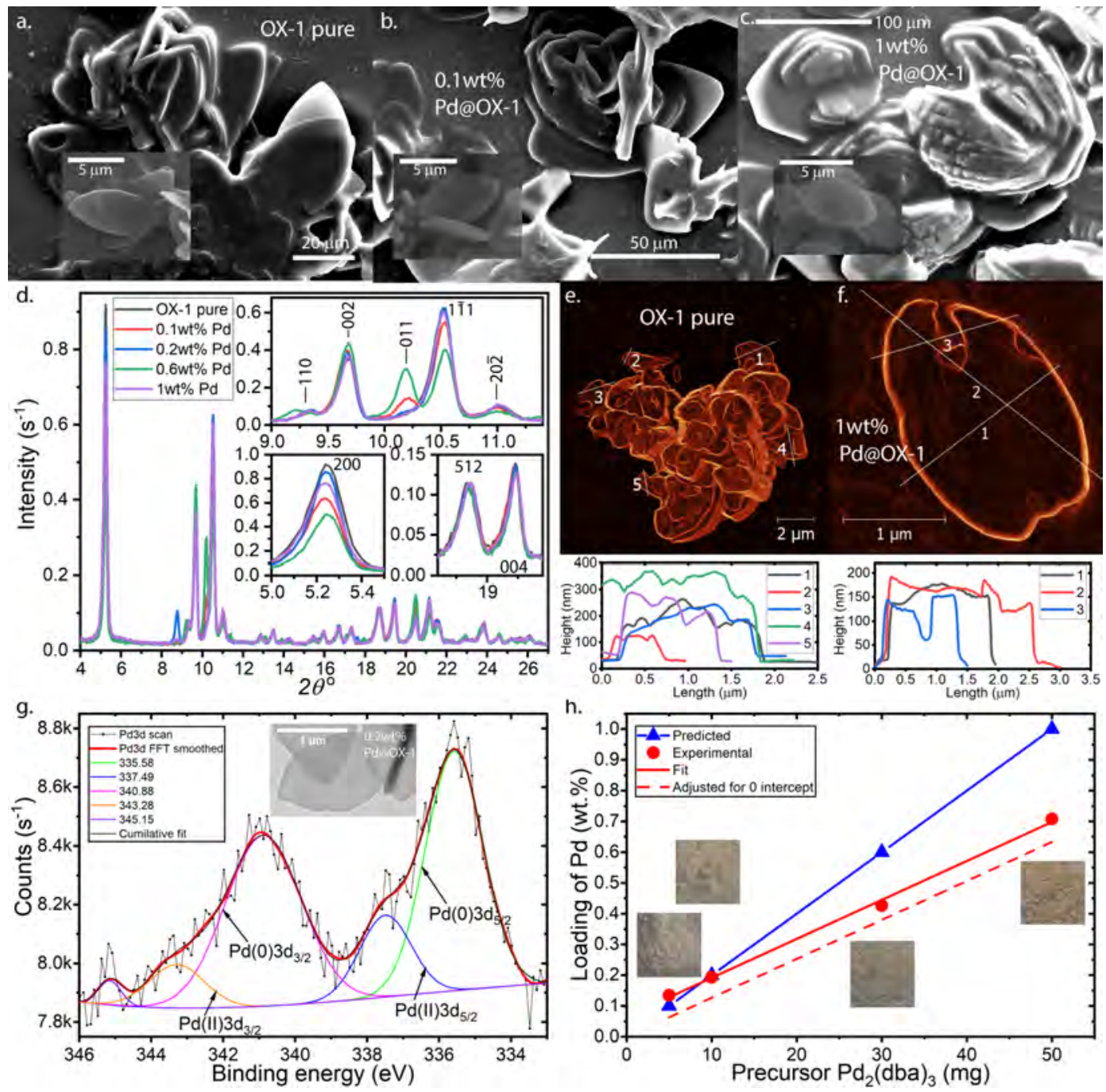

Figure 2. OX-1 and Pd@OX-1 crystals: SEM (a-c) and AFM images (e-f) of pure OX-1 (a,e) and Pd@OX-1 (b,c,f); PXRD patterns (d) of a series of Pd@OX-1 materials with different Pd loadings; XPS spectrum (g) of the 0.2 wt.\% Pd@OX-1 with a TEM image of the crystal inset; theoretical and experimental Pd content in Pd@OX-1 measured by XRF (h) for the same series of samples as (d), with photos of the respective powders.

components (see Figure 1(b)): two different Zn centres and three different orientations of BDC linkers. Type 1 linkers connect the metal nodes along the $\mathrm{x}$-axis and type 2 linkers connect the metal nodes along the y-axis - together they create the 2D layers of the structure. Type 3 linkers act as spacers and cross-bracing between the 2D layers. Each metal node (see Figure 1(c)) is made of three $\mathrm{Zn}$ centres, to which eight BDC linkers are coordinated. Two of the $\mathrm{Zn}$ centres have tetrahedral coordination environments and one has a square planar coordination environment.

OX-1 is a highly attractive material for practical applications: the rapid high yield synthesis route (detailed above) is readily scaled - while the nominal reported yield is about $500 \mathrm{mg}$, as much as $3 \mathrm{~g}$ of $\mathrm{OX}-1$ powder was obtained from a single reaction as part of this work with an insignificant increase in processing time. Moreover, OX-1 is readily functionalised: guest species can be incorporated via the same single pot method. The possibility of pelletising OX-1, and its functionalised variants, without loss of crystallinity, is also shown in this work, making it even more practicable (see specular reflection IR methods in SI).

\section{Capture of Pd onto OX-1 active sites}

The chemical reaction described above produced leaf-shaped crystals with sizes varying between 5 and $100 \mu \mathrm{m}$ in length and between $100 \mathrm{~nm}$ and $2 \mu \mathrm{m}$ in thickness. Figure 2(a-c) shows SEM images of pure OX-1 crystals and Pd@OX-1 crystals, while Figure 2(e-f) shows AFM images with an edge detection filter applied and some extracted height profiles. These show that the smaller crystals do not change morphology when increasing amounts of precursor $\mathrm{Pd}_{2}(\mathrm{dba})_{3}$ complex are added to the gel synthesis. The larger crystals, however, undergo a change: Figure 2(c) shows that the shape of the leaves changes to a more hexagonal geometry and additional smaller layers are seen appearing on top of the large plates upon introduction of $50 \mathrm{mg}$ of $\mathrm{Pd}_{2}(\mathrm{dba})_{3}$ into the reaction gel. These changes in morphology are captured by X-ray diffraction (XRD) patterns shown in Figure 2(d). The numbers of Bragg reflections between various crystallographic planes changes, seen most strikingly in the changing magnitude of the $5.25^{\circ}$ peak corresponding to the (200) crystallographic planes, but the distances between planes do not change 
a.

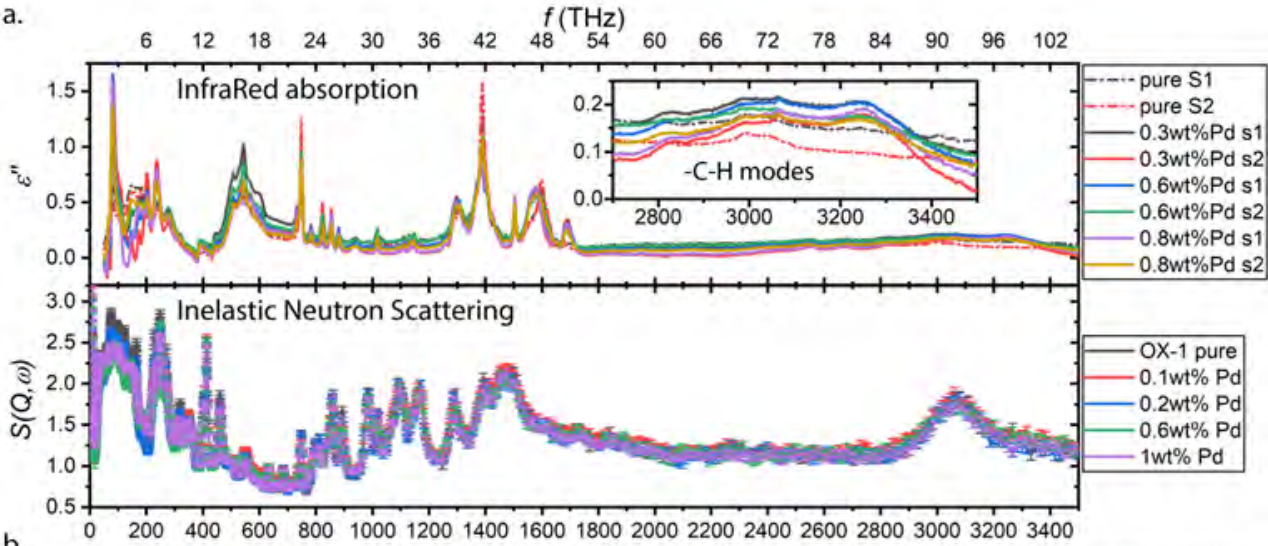

b.
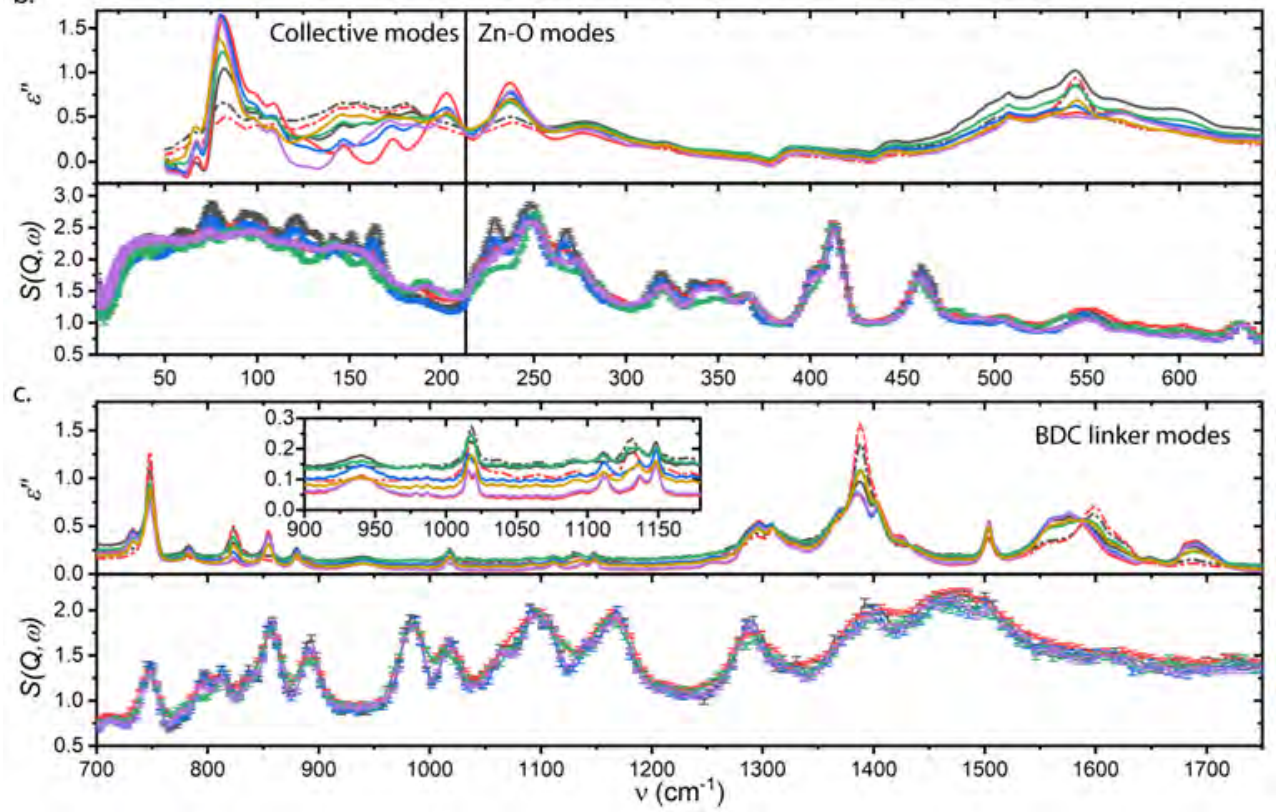

Figure 3. Infrared absorption spectra (top of each panel) and inelastic neutron scattering spectra (bottom of each panel) of Pd@OX-1 materials with various Pd loadings. These measurements were carried out on two separately synthesised series of materials as part of two separate beam times.

because the peaks do not shift to higher or lower angles. The presence of $\mathrm{Pd}$ thus does not distort the underlying structure of host OX-1 crystals, but the presence of $\mathrm{Pd}_{2}(\mathrm{dba})_{3}$ in the gel during synthesis affects their morphology. X-ray photoelectron spectroscopy (XPS) measurement (Figure 2(g)) of $0.2 \mathrm{wt} \%$ Pd@OX-1 (used for all catalysis experiments in this study) reveals the predominance of $\operatorname{Pd}(0)$ phase of palladium in the material. X-ray fluorescence spectroscopy (XRF) measurements (Figure 2(h)) of a series of variously loaded Pd@OX-1 crystals reveal a linear relationship between the amount of $\mathrm{Pd}_{2}(\mathrm{dba})_{3}$ added to the reaction gel and the amount of $\mathrm{Pd}$ detected in the washed and dried product.

Figure 3 shows infrared (IR) absorption spectra derived from specular reflectance measurements of pelletised $\mathrm{Pd} @ \mathrm{OX}-1$ samples via a Kramers-Kronig transformation. ${ }^{48}$ This method of IR spectroscopy provides information on IR active vibrational modes of compounds near the surface of the samples, with depth of penetration of IR beam being material dependent and not easily determined. These IR measurements were performed at the MIRIAM (B22) beamline of the Diamond Light Source synchrotron. Figure 3 also shows inelastic neutron scattering (INS) spectra taken at the TOSCA beamline of ISIS neutron source. INS is a highly penetrating non-selective technique, which provides information on all vibrational modes of the sample (no optical selection rule), probing the whole volume of the material and thus averaging across all local environments and especially sensitive to modes involving hydrogen motions.

The modes probed in IR absorption spectra are all detected in INS spectra, but the relative intensities of those peaks do not match. Furthermore, INS spectra also contain IR inactive modes. The spectra for the pristine OX-1 material thus resemble each other but do not match exactly, which might be due to the different penetration depth and selection rules for the two techniques. When $\mathrm{Pd}$ is introduced, dramatically different changes are observed by the two methods, which emphasises the complementarity of IR and INS spectroscopies.

$A b$ initio density functional theory (DFT) calculations for a very similar material, MOF-5, were reported by Civalleri and co-workers $^{49}$ and can serve as a rough guide for vibrational mode assignment in spectra for OX-1. As indicated in Figure 3, collective modes of the framework are present below $200 \mathrm{~cm}^{-1}$, various $\mathrm{Zn}-\mathrm{O}$ bond modes appear between 200 and $650 \mathrm{~cm}^{-1}$, and the various modes of BDC linkers, or their components, appear above $700 \mathrm{~cm}^{-1}$. Both IR and INS spectra show 

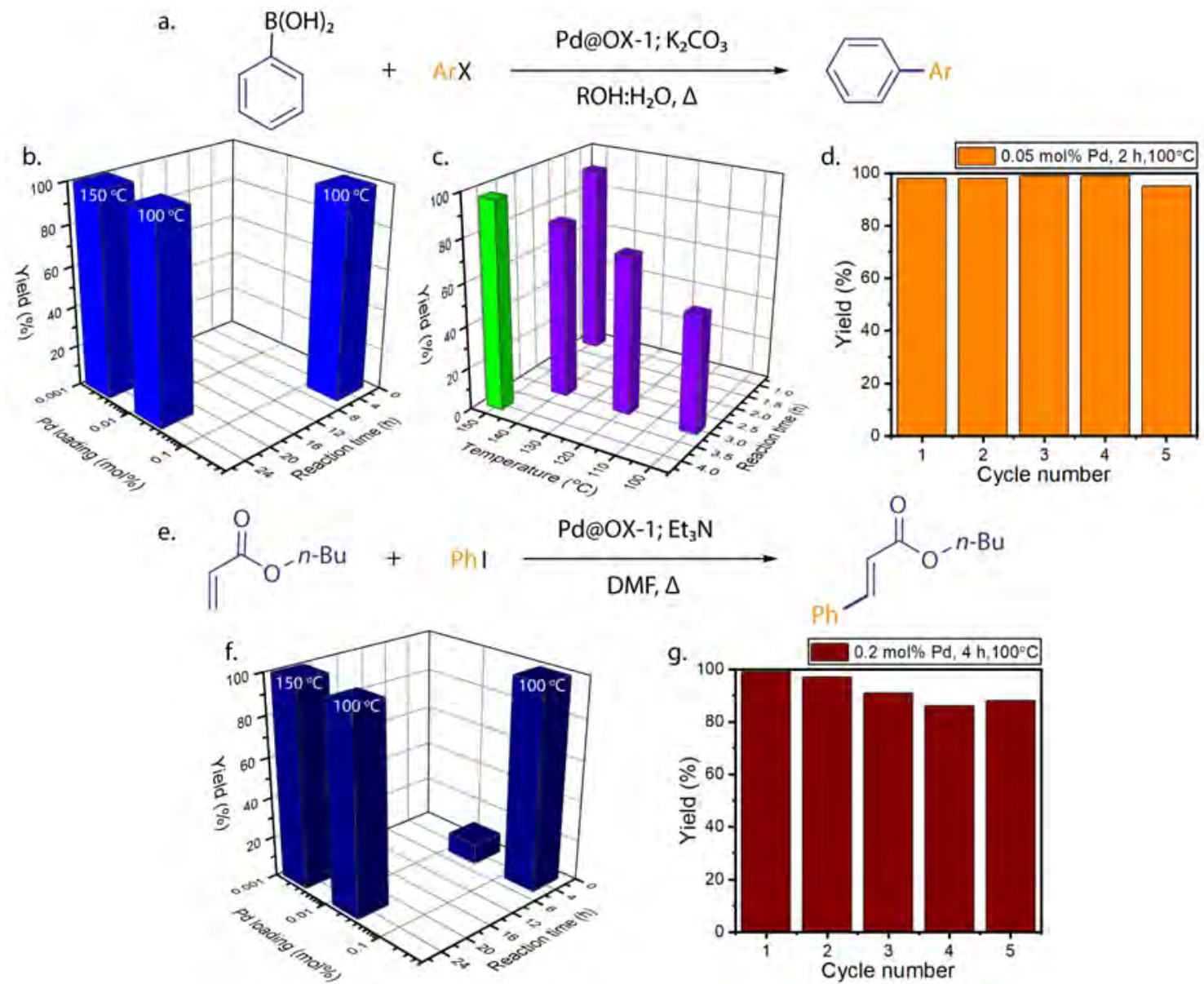

Figure 4. Pd@OX-1 catalytic performance in the Suzuki and Heck reactions. Model Suzuki reaction (a); variation of Pd loading for the reaction, $\mathrm{ArX}=\mathrm{PhI}, \mathrm{ROH}=\mathrm{MeOH}(\mathrm{b})$; optimization of the reaction conditions, $\mathrm{ArX}=4$-bromoanisole with $0.002 \mathrm{~mol} \% \mathrm{of} \mathrm{Pd}, \mathrm{ROH}=$ $i$-PrOH (c); recycling of the material, $\mathrm{ArX}=\mathrm{PhI}$ with $0.05 \mathrm{~mol} \%$ of $\mathrm{Pd}$ at $100{ }^{\circ} \mathrm{C}, \mathrm{ROH}=i$ - $\mathrm{PrOH}(\mathrm{d})$. Model Heck reaction (e); variation of $\mathrm{Pd}$ loading (f); recycling of the material, $0.2 \mathrm{~mol} \%$ of $\mathrm{Pd}$ at $100{ }^{\circ} \mathrm{C}(\mathrm{g})$. This data is given in table in the supporting information.

significant changes in collective modes and $\mathrm{Zn}-\mathrm{O}$ bond modes, but only IR spectra show noticeable changes in the BDC linker modes. Closer examination also reveals that the changes below $700 \mathrm{~cm}^{-1}$ are different for IR and INS spectra. IR reveals a dramatic enhancement of the collective mode at $80 \mathrm{~cm}^{-1}$, and noticeable enhancement of bands at 200 and $280 \mathrm{~cm}^{-1}$. INS spectra, on the other hand, show dampening of most collective modes up to $175 \mathrm{~cm}^{-1}$, as well as the sidebands of the triple peak in the region 213-280 $\mathrm{cm}^{-1}$. The BDC linker modes are also affected differently. IR spectra show dramatic changes in practically all BDC modes, whereas INS spectra show minor changes, which are difficult to attribute to introduction of $\mathrm{Pd}$ in the sample. Moreover, none of the changes in IR spectra appear to scale with amount of Pd loaded into the samples. This is most clearly seen in the shift of peaks from around $1600 \mathrm{~cm}^{-1}$ down to about $1575 \mathrm{~cm}^{-1}$ : all studied loadings appear to have the same step-like effect. However, the changes in INS spectra appear to follow the same trend as the changes in magnitudes of XRD peaks, most notably the (200) peak, shown in Figure 2(d). The significantly lower frequency $\left(<700 \mathrm{~cm}^{-1}\right)$ changes in INS spectra can be attributed to the changes in morphology of the crystals discussed above. These INS spectra therefore suggest that most modes in $\mathrm{Pd} @ \mathrm{OX}-1$ composites are not significantly affected in the presence of Pd. The IR spectra, however, identify the BDC linkers of OX-1 as primary interaction sites between $\mathrm{Pd}$ guests and the host framework. The BDC linkers are thus the most likely active sites for attachment of Pd species in the Pd@OX-1 composite system.

\section{Catalytic performance of guest $\mathrm{Pd}$ species}

Catalytic performance of 0.2 wt $\%$ Pd@OX-1 was studied in the Suzuki cross-coupling and the Heck arylation reactions, as they are well known to be efficiently catalysed by multiple palladium species. ${ }^{26,27,29}$ All catalytic studies were performed under air in order to examine the robustness of the material.

In the Suzuki coupling of phenylboronic acid with iodobenzene (Figure 4(a)) catalyst activity was studied by decreasing the Pd loading from $0.2 \mathrm{~mol} \%$ down to $0.002 \mathrm{~mol} \%$, where the latter corresponds to $20 \mathrm{ppm}$ loading relative to $\mathrm{PhI}$. The reaction was conducted in $i$-PrOH: $\mathrm{H}_{2} \mathrm{O}(1: 1)$ media and the product formation was analysed by GC-MS. In all three cases $>99 \%$ quantitative yields of biphenyl were reached (Figure 4(b)). Because of the high yields with PhI we used Ar-X, which is of lower reactivity, to address the material activity: 4bromoanisole with $20 \mathrm{ppm}$ catalyst loading. After 3 hours of the reaction at $100{ }^{\circ} \mathrm{C}, 120^{\circ} \mathrm{C}$, and $140{ }^{\circ} \mathrm{C}$ the product yield grew from $55 \%$ up to $82 \%$. Moreover at $150{ }^{\circ} \mathrm{C} 91 \%$ yield was achieved already after 1 hour, and after 4 hours reached $97 \%$ of the 4-methoxybiphenyl product (Figure 4(c)). Following these results, preliminary scope studies were conducted, using $0.05 \mathrm{~mol} \%$ of $\mathrm{Pd}$ at $100^{\circ} \mathrm{C}$. Both aryl iodides and bromides 

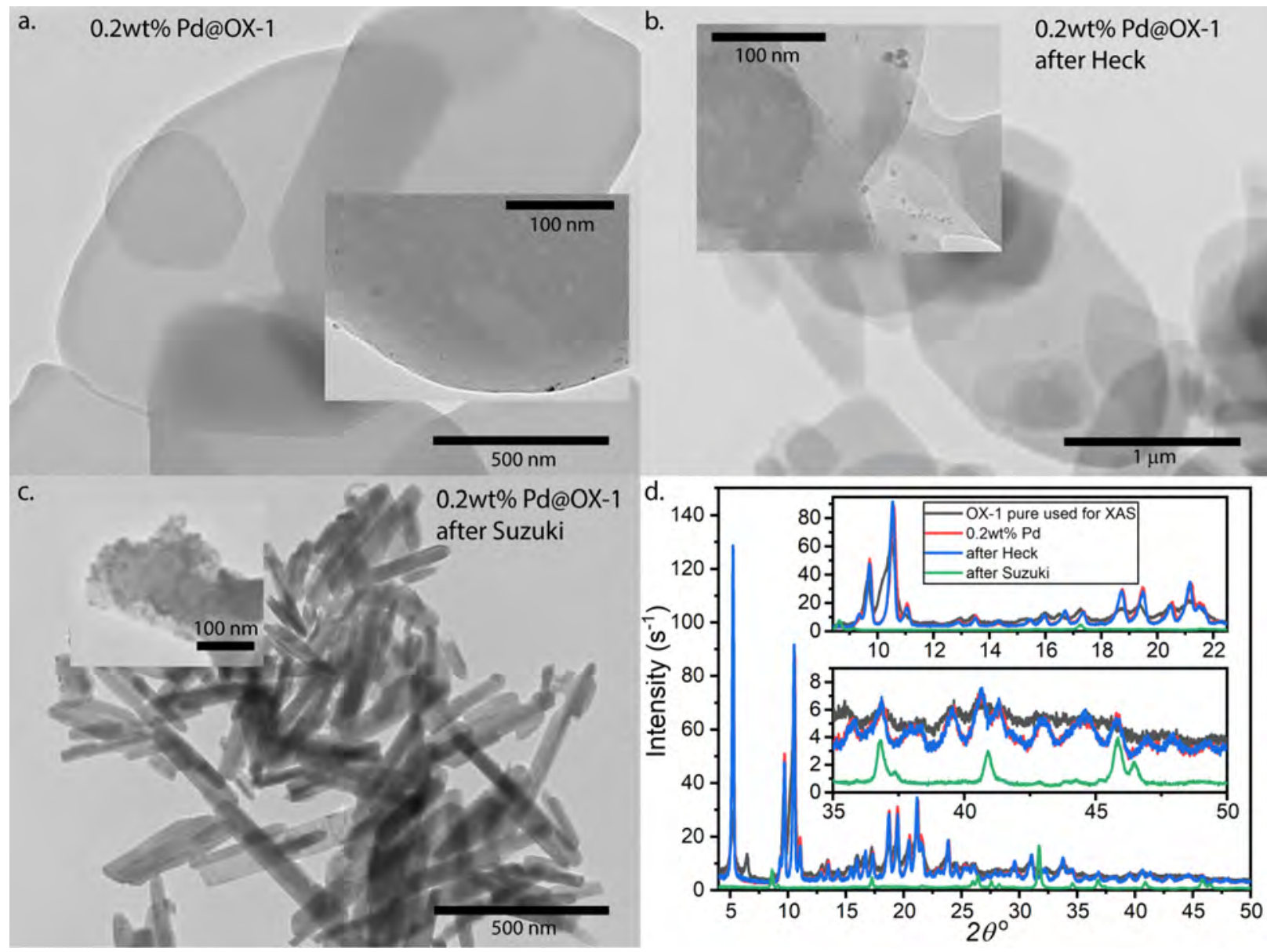

Figure 5. TEM images of (a) as synthesised Pd@OX-1 crystals and the same crystals after the (b) Heck and (c) Suzuki reactions. (d) XRD patterns for of the same set of samples.

gave yields from good to excellent (see the SI). Electron properties of the substituents in aryl halide or aryl boronic acid have no significant effect on the outcome of the reaction. Steric bulkiness provided by orto-substituent (2-iodobenzaldehide) also did not affect an excellent product yield.

Even though visually the microstructure of the catalyst material changed after the reaction, seeming to be more agglomerated, we have analysed the possibility of catalyst recycling with $\mathrm{PhI}$, which is usually difficult because the freed iodide species in the solution are known to poison heterogeneous catalysts. The reaction was stopped after 2 hours ( $98 \%$ yield), material was centrifuged, washed and re-used for another four cycles without significant loss of activity (see Figure 4(d)).

Next, the catalyst behaviour in the Heck reaction was studied (Figure 4(e)). The approach was similar, butyl acrylate arylation with $\mathrm{PhI}$ was investigated with decreasing Pd loading. The outcome was roughly the same as for the Suzuki coupling. Butyl cinnamate was obtained with $>99 \%$ yield after 24 hours at $150^{\circ} \mathrm{C}$ with only $20 \mathrm{ppm}$ of Pd (Figure 4(f)). Although the catalyst was active towards iodide, when switching to 4-bromoanisole only traces of the reaction product were detected after 24 hours at an elevated temperature of $180{ }^{\circ} \mathrm{C}$ for either butyl acrylate or styrene. However, as with Suzuki coupling, the material could be recycled for five times, showing only slight loss of activity for PhI conversion (Figure $4(\mathrm{~g})$ ). Even though the medium in which the Heck reaction is performed is strikingly similar to the medium in which $\mathrm{Pd} @ \mathrm{OX}-1$ is synthesized $\left(\mathrm{DMF}, \mathrm{Et}_{3} \mathrm{~N}\right)$, the MOF appeared visually unchanged after the reaction except a slight change in colour. Substrate scope was evaluated for the Heck reaction. Yields from good to excellent were obtained for all substrates (see the SI) with either electron withdrawing or electron donating groups. Utilisation of an orto-substituted iodotoluene resulted in lower yield of $81 \%$. Comparing different acrylates we have observed that longer chain provides higher yield (Me$-84 \%$, Et- $-87 \%, n$-Bu- $-98 \%$ ); while with increasing steric bulkiness the yield of the product was slightly lowered $(t$-Bu- $92 \%)$.

This ensemble of results demonstrates the high catalytic activity of Pd@OX-1 in a broad variety of conditions, as well as retainment of catalytic properties after both the Suzuki coupling and the Heck reactions.

\section{Local structure and persistence of active sites}

In recent decades diverse catalytic applications of $\mathrm{Pd}$ nanoparticles in cross-coupling reactions were facilitated by release of highly active $\mathrm{Pd}$ species to solution via leaching. ${ }^{19}$ However, leaching of $\mathrm{Pd}$ species to solution often leads to dramatic loss of catalytic activity on the next cycle due to metal losses. Re-capture of metal species from solution back to the surface of support after completing the catalytic process is the key challenge for retaining activity and avoiding contamination with palladium traces. Support materials do not routinely 

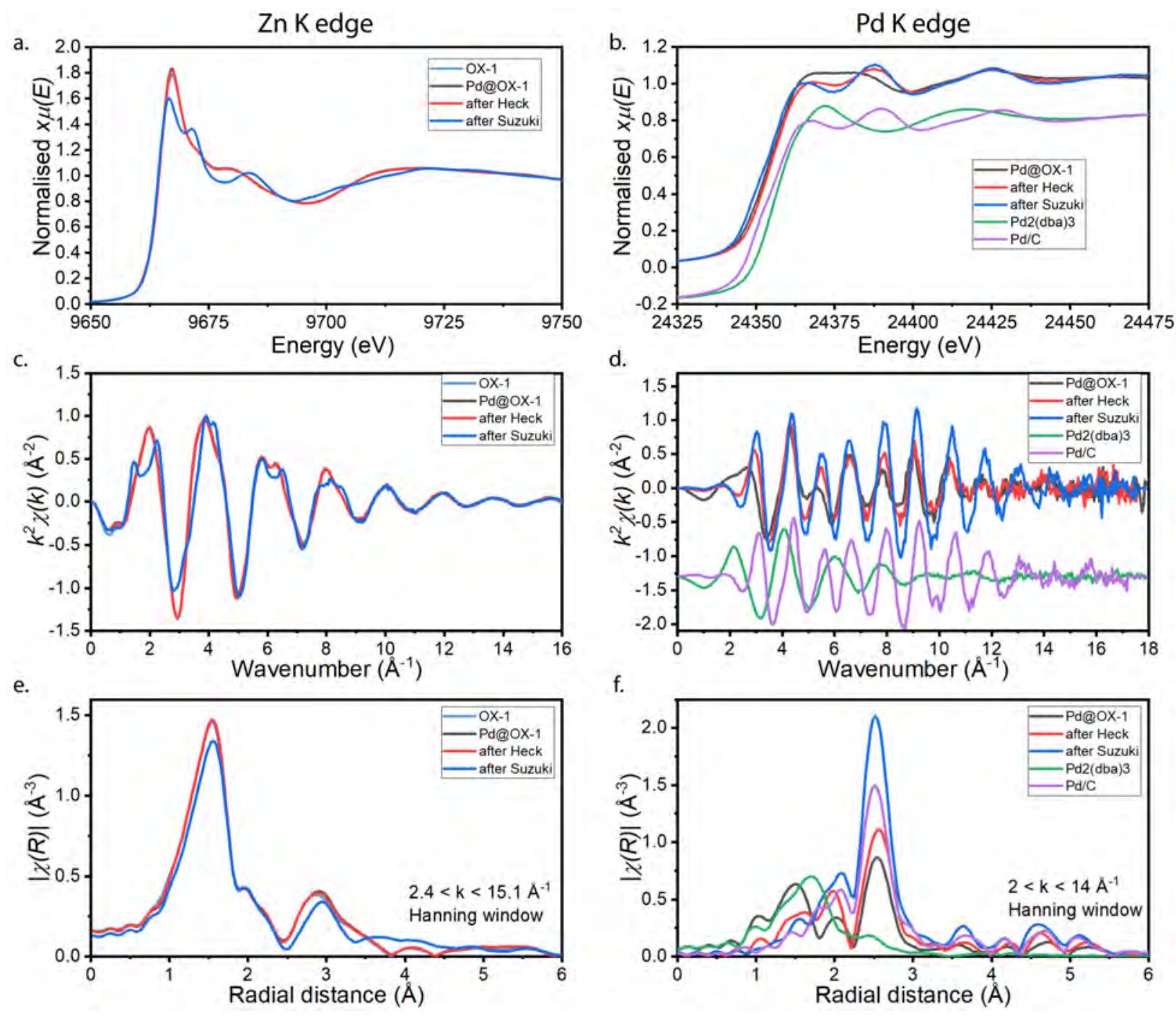

Figure 6. X-ray absorption spectra at the Zn (a,c,e) and Pd (b,d,f) K-edge of pure OX-1, pristine 0.2 wt $\%$ Pd@OX-1, Pd@OX-1 after the Heck and Suzuki reactions, of $\mathrm{Pd}_{2}(\mathrm{dba})_{3}$ crystals and of Pd nanoparticles on graphite. In panels $(\mathrm{b})$ and $(\mathrm{d})$ the spectra of $\mathrm{Pd} 2(\mathrm{dba})_{3}$ and $\mathrm{Pd} / \mathrm{C}$ have been shifted vertically for sake of comparison. Data processing and fitting were done in Demeter. ${ }^{50}$

retain ability to re-capture metal species from solution after catalytic transformation under harsh conditions, causing significant reconstruction of surface. Thus, the observed performance of the material developed here prompted us to conduct more detailed characterization.

Pd@OX-1 owes its good catalytic performance and the possibility of recycling the material to the structure and persistence of its BDC active sites for Pd attachment. These are studied by probing the structure and local environments of $\mathrm{Zn}$ centres of OX-1 and of the various Pd species.

TEM imaging detects the presence of fine scale $\mathrm{Pd}$ nanoparticles on as synthesised 0.2 wt $\% \mathrm{Pd} @ \mathrm{OX}-1$ crystals (Figure 5(a)) with a size range of $c a$. 2-5 nm. Smaller particles were not detected at this microscope's resolution. After the Heck reaction (see SI for reaction details) the morphology of the crystals appears to be unchanged, but most of the Pd nanoparticles appear to have grown in size, with particles as large as $10 \mathrm{~nm}$ (Figure 5(b)). After the Suzuki reaction (see SI for reaction details), however, the shape of the recovered crystals changes dramatically to oblong particles. The size of Pd nanoparticles became even larger than in the case of the Heck reaction reaching $15-20 \mathrm{~nm}$, are seen in TEM images
(Figure 5(c)). XRD measurements (Figure 5(d)) reveal that crystal structure of $\mathrm{Pd} @ \mathrm{OX}-1$ does not change during the Heck reaction, but is completely different after the Suzuki reaction. This is remarkable: not only can OX-1 survive in DMF at $100^{\circ} \mathrm{C}$ for at least 4 hours for the Heck reaction and recapture ${ }^{26,51-55}$ the active Pd species, but even after a dramatic change in microstructure during the Suzuki reaction it retains the $\mathrm{Pd}$ species and is still active in following cycles. To the best of our knowledge this is a rare example of $\mathrm{Pd}$ recapture after total reconstruction of the material.

X-ray Absorption Spectroscopy (XAS) experiments were performed at the B18 beamline ${ }^{56}$ of Diamond Light Source to study the possible changes in OX-1 crystal structure, as well as the structure and location of the attached Pd species, with results shown in Figure 6. X-ray Absorption Near Edge Structure (XANES) and Extended X-ray Absorption Fine Structure (EXAFS) data were collected at the $\mathrm{Zn}$ and Pd K-edges for the $0.2 \mathrm{wt} \% \mathrm{Pd} @ \mathrm{OX}-1$ as synthesised sample, a sample that was used to catalyse the Heck reaction, and a sample that was used to catalyse the Suzuki reaction. Moreover, precursor $\mathrm{Pd}_{2}(\mathrm{dba})_{3}$ crystals and a sample of $\mathrm{Pd}$ nanoparticles on graphite ${ }^{57}$ derived from $\mathrm{Pd}_{2}(\mathrm{dba})_{3}$ were 

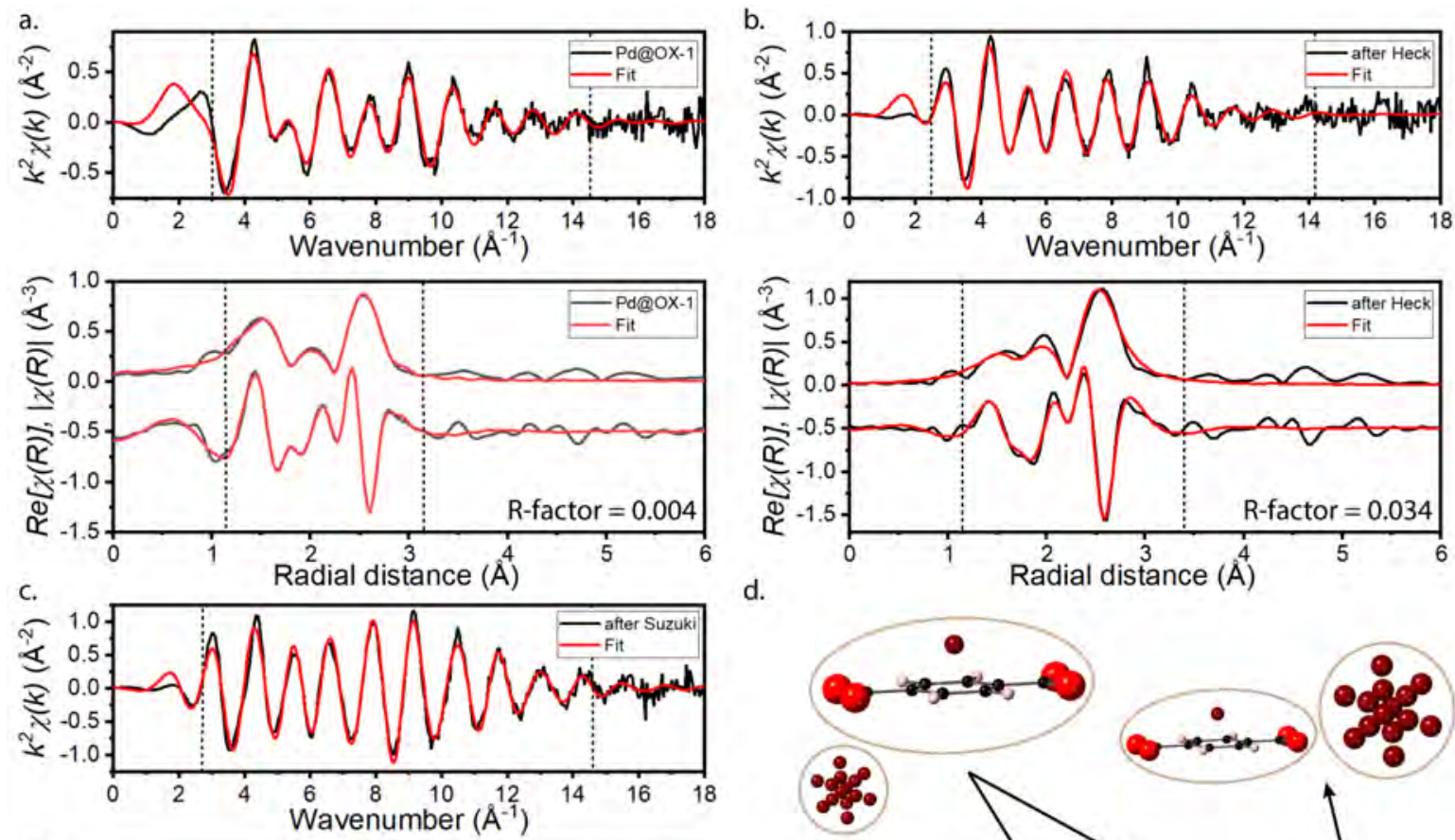

d.

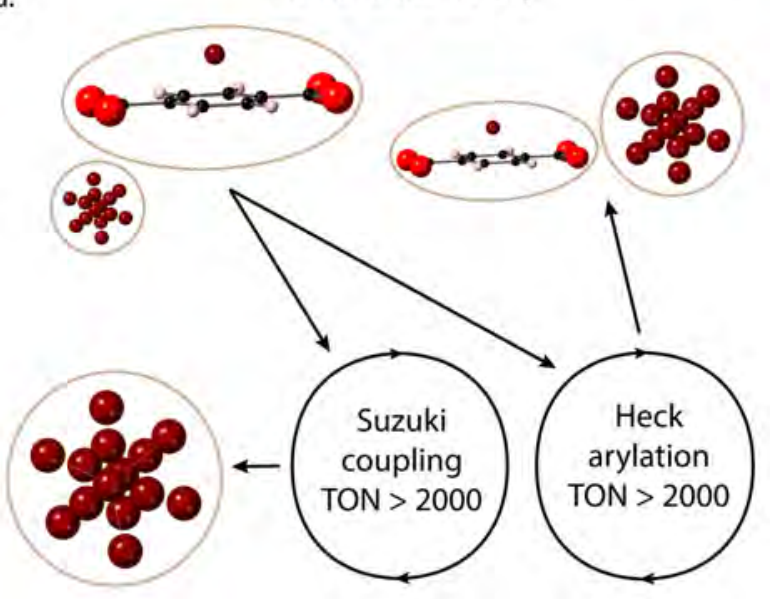

Figure 7. Fitting of EXAFS spectra for the as synthesised (a) 0.2 wt.\% Pd@OX-1, (b) Pd@OX-1 after the Heck reaction and (c) Pd@OX-1 after the Suzuki reaction using models of Pd environments given in (d). The "Turn Over Number" (TON) is defined as the number of reactions catalysed per Pd atom. Data processing and fitting were done in Demeter. ${ }^{50}$

measured at the Pd K-edge for reference. This XAS data reflects the local structure around $\mathrm{Zn}$ and $\mathrm{Pd}$ atoms in the Pd@OX-1 system.

$\mathrm{Zn}$ K-edge measurments reveal that the introduction of $\mathrm{Pd}_{2}(\mathrm{dba})_{3}$ into the synthesis does not change the framework structure around the $\mathrm{Zn}$ centers of OX-1. The spectra for OX-1 and $\mathrm{Pd} @ \mathrm{OX}-1$ coincide almost exactly. There is only a slight increase of the white-line intensity when loading Pd into the host framework. This can be ascribed to a subtle enhancement of the localisation of the final electronic states of $\mathrm{Zn}$. This difference is negligible in the EXAFS signal. Thus the local structure around $\mathrm{Zn}$ can be considered not affected by the loading of the Pd.

$\mathrm{Zn} \mathrm{K}$-edge data further shows that the $\mathrm{Zn}$ environment of Pd@OX-1 does not change at all during the Heck reaction, confirming TEM and XRD observations discussed above. During the Suzuki reaction, however, the $\mathrm{Zn}$ environment is significantly altered: the profile of the XANES changes drastically becoming more structured. On the other hand, the pseudo radial distribution function of the sample after Suzuki reaction, in particular, the first coordination shell is similar to the one obtained for the pristine Pd@OX-1 sample, thus the short range order appears to be preserved. The changes in the XANES may be due to distortions in the bond angles which influences mainly the multiple scattering contributions. Indeed, the EXAFS signal for the sample after Suzuki reaction is more complex than the one for the original compound. This again confirms TEM and XRD observations, and identifies this new structure as a new MOF-type material with long range periodicity with a consistent local enviroment.

Pd K-edge data, Figure 6(b,d,f), shows that the XANES of the samples after catalytic reactions are similar to the one obtained from the pristine Pd@OX-1 sample. The only difference is that the broad white-line of the pristine sample, characteristic of low order environments, splits into two structures after the reaction. This XANES profile with the two structures is very similar to the one obtained for $\mathrm{Pd} / \mathrm{C}$ sample which agrees with the fact of having larger $\mathrm{Pd}$ nanoparticles than the pristine sample.

The model used for performing the fitting analysis of the EXAFS data considered the $\mathrm{Pd}-\mathrm{C}$ scattering path to the carbon atoms of benzene rings on BDC linkers, where $\mathrm{Pd}$ is assumed to be equidistant from all six members of the ring, and the Pd$\mathrm{Pd}$ scattering paths characteristic of the face-centred cubic (FCC) Pd bulk. The fits can be seen in Figure 7 and the results from the fit can be found in Table S3 of the SI. The model seems to agree reasonably well with the experimental data despite its simplicity. From the fit we obtain a very low coordination number of Pd in as synthesised Pd@OX-1, which suggests that the clusters of Pd inside the composite are very small, reflecting that a very high number of Pd atoms belong to surfaces of Pd particles. We reasoned that the as-synthesised Pd@OX-1 contains atomic $\mathrm{Pd}$ and $\mathrm{Pd}$ in small clusters coordinated to the $\pi$-system of the benzene rings of BDC linkers. Only a small number of particles are as big as those seen in the TEM images, while most of Pd in the sample is not resolved. After both catalytic reactions, the coordination number of the $\mathrm{Pd}-\mathrm{Pd}$ 
contribution increases, which indicates that the size of $\mathrm{Pd}$ nanoparticles has grown, in agreement with the TEM images. In the case of the after Suzuki sample in particular, the contribudtion of the Pd on ligand component of the model was found to be negligible, so it was excluded from the fit.

Some recent results ${ }^{21,22}$ also show that a shift to lower energy and a sharpening of the first near-edge peak of the XANES region are indicative of palladium hydride formation. In addition, this hydrogen bonding is accompanied by an elongation of the Pd-Pd distance (see Table S3). It is thus possible that during the Suzuki reaction some heteroatom insertion of hydrogen into the Pd nanoparticles may take place.

\section{CONCLUSIONS}

This paper presents a method of rapid in situ functionalisation of OX-1 metal-organic nanosheets with catalytically active $\mathrm{Pd}$ species, obtained via a one-step one-pot supramolecular synthesis route. The loading of $\mathrm{Pd}$ can be controlled by adjusting the amount of $\operatorname{Pd}_{2}(\mathrm{dba})_{3}$ added to the synthesis. The resulting Pd@OX-1 composite crystals contain predominantly atomic and small cluster Pd species, which selectively reside on benzene rings of the BDC linkers. The composites are shown to efficiently catalyze the Suzuki coupling and the Heck arylation reactions under a variety of conditions. The material retains the ability to recapture active $\mathrm{Pd}$ species during the reactions, which is not a routine property of common substrates for $\mathrm{Pd}$ nanoparticles. This enables the demonstrated potential for $\mathrm{Pd} @ \mathrm{OX}-1$ to be recycled in reaction cycles of either type without loss of activity. More detailed studies are warranted to fully explore catalytic potential of the developed material as follow-on work, to systematically investigate the influence of various reaction conditions and substrates, amongst others. Undoubtedly, with its variety of Pd species and tunability of $\mathrm{Pd}$ loading, Pd@OX-1 shows promise as an attractive system for further study, with potential industrial applications in organic synthesis.

\section{ASSOCIATED CONTENT}

\section{Supporting Information}

The Supporting Information is available free of charge on the ACS Publications website. Experimental methods, additional XRD data, IR spectroscopy dielectric functions and X-ray absorption spectroscopy analysis.

\section{AUTHOR INFORMATION}

\section{Corresponding Author}

* V.P. Ananikov, email: val@ioc.ac.ru

* J.C. Tan, email: jin-chong.tan@eng.ox.ac.uk

\section{Author Contributions}

The manuscript was written through contributions of all authors. All authors have given approval to the final version of the manuscript. $\$$ These authors contributed equally.

\section{Funding Sources}

The research in the MMC Lab (J.C.T.) was supported by the ERC Consolidator Grant - PROMOFS under the grant agreement 771575, and the EPSRC grant no. EP/N014960/1. Catalytic performance investigation (V.P.A.) was supported by the Russian Science Foundation (RSF Grant 14-50-00126).

\section{ACKNOWLEDGMENTS}

We acknowledge the Diamond Light Source for beamtime no. SM14902 at B22 MIRIAM, and beamtime no. SP17146 in B18. We thank the ISIS facility for the provision of neutron beamtime no. RB1810348 on the TOSCA spectrometer. We are grateful to the ISIS Rutherford Appleton Laboratory, especially Dr. Gavin Stenning and Dr Marek Jura at the R53 Materials Characterization Laboratory for access to XRF and XRD. We thank the Research Complex at Harwell $(\mathrm{RCaH})$ for providing access to materials characterization facilities.

\section{REFERENCES}

1. Hendon, C. H.; Rieth, A. J.; Korzynski, M. D.; Dinca, M., Grand Challenges and Future Opportunities for Metal-Organic Frameworks. ACS Cent. Sci. 2017, 3 (6), 554-563, DOI 10.1021/acscentsci.7b00197.

2. Dolgopolova, E. A.; Shustova, N. B., Metal-organic framework photophysics: Optoelectronic devices, photoswitches, sensors, and photocatalysts. MRS Bull. 2016, 41 (11), 890-895, DOI 10.1557/mrs.2016.246.

3. Zhu, L.; Liu, X. Q.; Jiang, H. L.; Sun, L. B., Metal-Organic Frameworks for Heterogeneous Basic Catalysis. Chem. Rev. 2017, 117 (12), 8129-8176, DOI 10.1021/acs.chemrev.7b00091.

4. $\quad$ Chughtai, A. H.; Ahmad, N.; Younus, H. A.; Laypkov, A.; Verpoort, F., Metal-organic frameworks: versatile heterogeneous catalysts for efficient catalytic organic transformations. Chem. Soc. Rev. 2015, 44 (19), 6804-6852, DOI 10.1039/c4cs00395k.

5. Lee, J.; Farha, O. K.; Roberts, J.; Scheidt, K. A.; Nguyen, S. T.; Hupp, J. T., Metal-organic framework materials as catalysts. Chem. Soc. Rev. 2009, 38 (5), 1450-1460, DOI 10.1039/b807080f.

6. Gascon, J.; Corma, A.; Kapteijn, F.; Llabrés i Xamena, F. X., Metal Organic Framework Catalysis: Quo vadis? ACS Catal. 2013, 4 (2), 361-378, DOI 10.1021/cs400959k

7. Vermoortele, F.; Vandichel, M.; Van de Voorde, B.; Ameloot, R.; Waroquier, M.; Van Speybroeck, V.; De Vos, D. E., Electronic effects of linker substitution on Lewis acid catalysis with metal-organic frameworks. Angew. Chem. Int. Ed. 2012, 51 (20), 48874890, DOI 10.1002/anie.201108565.

8. Vermoortele, F.; Ameloot, R.; Alaerts, L.; Matthessen, R.; Carlier, B.; Fernandez, E. V. R.; Gascon, J.; Kapteijn, F.; De Vos, D. E., Tuning the catalytic performance of metal-organic frameworks in fine chemistry by active site engineering. J. Mater. Chem. 2012, 22 (20), 10313-10321, DOI 10.1039/c2jm16030g.

9. Zhu, Q. L.; Xu, Q., Immobilization of Ultrafine Metal Nanoparticles to High-Surface-Area Materials and Their Catalytic Applications. Chem 2016, 1 (2), 220-245, DOI 10.1016/j.chempr.2016.07.005.

10. Mehta, J. P.; Tian, T.; Zeng, Z. X.; Divitini, G.; Connolly, B. M.; Midgley, P. A.; Tan, J. C.; Fairen-Jimenez, D.; Wheatley, A. E. H., Sol-Gel Synthesis of Robust Metal-Organic Frameworks for Nanoparticle Encapsulation. Adv. Funct. Mater. 2018, 28 (8), e1705588, DOI 10.1002/adfm.201705588.

11. Zhao, M.; Lu, Q.; Ma, Q.; Zhang, H., Two-Dimensional Metal-Organic Framework Nanosheets. Small Methods 2017, 1 (1-2), 1600030, DOI 10.1002/smtd.201600030.

12. Pustovarenko, A.; Goesten, M. G.; Sachdeva, S.; Shan, M.; Amghouz, Z.; Belmabkhout, Y.; Dikhtiarenko, A.; Rodenas, T.; Keskin, D.; Voets, I. K.; Weckhuysen, B. M.; Eddaoudi, M.; de Smet, L.; Sudholter, E. J. R.; Kapteijn, F.; Seoane, B.; Gascon, J., Nanosheets of Nonlayered Aluminum Metal-Organic Frameworks through a Surfactant-Assisted Method. Adv. Mater. 2018, 30 (26), e1707234, DOI 10.1002/adma.201707234.

13. Chaudhari, A. K.; Tan, J. C., Mechanochromic MOF nanoplates: spatial molecular isolation of light-emitting guests in a sodalite framework structure. Nanoscale 2018, 10 (8), 3953-3960, DOI $10.1039 / \mathrm{c} 7 \mathrm{nr} 09730 \mathrm{a}$

14. Osada, M.; Sasaki, T., Two-dimensional dielectric nanosheets: novel nanoelectronics from nanocrystal building blocks. Adv. Mater. 2012, 24 (2), 210-228, DOI 10.1002/adma.201103241.

15. Mas-Balleste, R.; Gomez-Navarro, C.; Gomez-Herrero, J.; Zamora, F., 2D materials: to graphene and beyond. Nanoscale 2011, 3 (1), 20-30, DOI 10.1039/c0nr00323a.

16. He, T.; Ni, B.; Zhang, S.; Gong, Y.; Wang, H.; Gu, L.; Zhuang, J.; Hu, W.; Wang, X., Ultrathin 2D Zirconium Metal-Organic 
Framework Nanosheets: Preparation and Application in Photocatalysis. Small 2018, 14 (16), e1703929, DOI 10.1002/smll.201703929.

17. Chaudhari, A. K.; Kim, H. J.; Han, I.; Tan, J. C., Optochemically Responsive 2D Nanosheets of a 3D Metal-Organic Framework Material. Adv. Mater. 2017, 29 (27), e1701463, DOI 10.1002/adma.201701463.

18. Allendorf, M. D.; Medishetty, R.; Fischer, R. A., Guest molecules as a design element for metal-organic frameworks. MRS Bull. 2016, 41 (11), 865-869, DOI 10.1557/mrs.2016.244.

19. Balanta, A.; Godard, C.; Claver, C., Pd nanoparticles for CC coupling reactions. Chem. Soc. Rev. 2011, 40 (10), 4973-5057, DOI $10.1039 / \mathrm{c} 1 \mathrm{cs} 15195 \mathrm{a}$

20. Trzeciak, A. M., Pd Nanoparticles for Coupling Reactions and Domino/Tandem Reactions. Wiley-VCH: Weinheim, Germany: 2016.

21. Bugaev, A. L.; Guda, A. A.; Lazzarini, A.; Lomachenko, K. A.; Groppo, E.; Pellegrini, R.; Piovano, A.; Emerich, H.; Soldatov, A. V.; Bugaev, L. A.; Dmitriev, V. P.; van Bokhoven, J. A.; Lamberti, C., In situ formation of hydrides and carbides in palladium catalyst: When XANES is better than EXAFS and XRD. Catal. Today 2017, 283, 119-126, DOI 10.1016/j.cattod.2016.02.065.

22. Bugaev, A. L.; Usoltsev, O. A.; Lazzarini, A.; Lomachenko, K. A.; Guda, A. A.; Pellegrini, R.; Carosso, M.; Vitillo, J. G.; Groppo, E.; van Bokhoven, J. A.; Soldatov, A. V.; Lamberti, C., Time-resolved operando studies of carbon supported $\mathrm{Pd}$ nanoparticles under hydrogenation reactions by X-ray diffraction and absorption. Faraday Discuss. 2018, 208 (0), 187-205, DOI $10.1039 / \mathrm{c} 7 \mathrm{fd} 00211 \mathrm{~d}$

23. Carosso, M.; Lazzarini, A.; Piovano, A.; Pellegrini, R.; Morandi, S.; Manzoli, M.; Vitillo, J. G.; Ruiz, M. J.; Lamberti, C.; Groppo, E., Looking for the active hydrogen species in a $5 \mathrm{wt} \% \mathrm{Pt} / \mathrm{C}$ catalyst: a challenge for inelastic neutron scattering. Faraday Discuss. 2018, 208 (0), 227-242, DOI 10.1039/c7fd00214a.

24. Frenkel, A. I.; Yevick, A.; Cooper, C.; Vasic, R., Modeling the structure and composition of nanoparticles by extended X-ray absorption fine-structure spectroscopy. Annu. Rev. Anal. Chem. 2011, 4, 23-39, DOI 10.1146/annurev-anchem-061010-113906.

25. Lopes, C. W.; Cerrillo, J. L.; Palomares, A. E.; Rey, F.; Agostini, G., An in situ XAS study of the activation of precursordependent Pd nanoparticles. Phys. Chem. Chem. Phys. 2018, 20 (18), 12700-12709, DOI 10.1039/c8cp00517f

26. Eremin, D. B.; Ananikov, V. P., Understanding active species in catalytic transformations: From molecular catalysis to nanoparticles, leaching, "Cocktails" of catalysts and dynamic systems. Coord. Chem. Rev. 2017, 346, 2-19, DOI 10.1016/j.ccr.2016.12.021.

27. Ananikov, V. P.; Beletskaya, I. P., Toward the Ideal Catalyst: From Atomic Centers to a "Cocktail" of Catalysts. Organometallics 2012, 31 (5), 1595-1604, DOI 10.1021/om201120n.

28. Fairlamb, I. J. S.; Lee, A. F., Fundamental Pd-0/Pd-II Redox Steps in Cross-coupling Reactions: Homogeneous, Hybrid Homogeneous-Heterogeneous to Heterogeneous Mechanistic Pathways for C-C Couplings. $C-H$ and $C-X$ Bond Functionalization: Transition Metal Mediation; RSC Catal. Ser. 2013, (11), 72-107, DOI 10.1039/9781849737166.

29. Kashin, A. S.; Ananikov, V. P., Catalytic C-C and Cheteroatom bond formation reactions: in situ generated or preformed catalysts? Complicated mechanistic picture behind well-known experimental procedures. J. Org. Chem. 2013, 78 (22), 11117-11125, DOI $10.1021 /$ jo $402038 \mathrm{p}$.

30. Trzeciak, A. M.; Ziółkowski, J. J., Monomolecular, nanosized and heterogenized palladium catalysts for the Heck reaction. Coord. Chem. Rev. 2007, 251 (9-10), 1281-1293, DOI 10.1016/j.ccr.2006.11.013

31. Ranocchiari, M.; van Bokhoven, J. A., Catalysis by metalorganic frameworks: fundamentals and opportunities. Phys. Chem. Chem. Phys. 2011, 13 (14), 6388-6396, DOI 10.1039/c0cp02394a.

32. Fortea-Perez, F. R.; Mon, M.; Ferrando-Soria, J.; Boronat, M.; Leyva-Perez, A.; Corma, A.; Herrera, J. M.; Osadchii, D. Gascon, J.; Armentano, D.; Pardo, E., The MOF-driven synthesis of supported palladium clusters with catalytic activity for carbene- mediated chemistry. Nat. Mater. 2017, 16 (7), 760-766, DOI 10.1038/nmat4910

33. Liu, J.; Ye, J.; Li, Z.; Otake, K. I.; Liao, Y.; Peters, A. W.; Noh, H.; Truhlar, D. G.; Gagliardi, L.; Cramer, C. J.; Farha, O. K.; Hupp, J. T., Beyond the Active Site: Tuning the Activity and Selectivity of a Metal-Organic Framework-Supported Ni Catalyst for Ethylene Dimerization. J. Am. Chem. Soc. 2018, 140 (36), 1117411178, DOI 10.1021/jacs.8b06006.

34. Pascanu, V.; Yao, Q.; Bermejo Gomez, A.; Gustafsson, M.; Yun, Y.; Wan, W.; Samain, L.; Zou, X.; Martin-Matute, B., Sustainable catalysis: rational Pd loading on MIL-101Cr-NH $\mathrm{NH}_{2}$ for more efficient and recyclable Suzuki-Miyaura reactions. Chem. - Eur. J. 2013, 19 (51), 17483-17493, DOI 10.1002/chem.201302621.

35. Metzger, E. D.; Brozek, C. K.; Comito, R. J.; Dincă, M., Selective Dimerization of Ethylene to 1-Butene with a Porous Catalyst ACS Cent. Sci. 2016, 2 (3), 148-153, DOI 10.1021/acscentsci.6b00012.

36. Pascanu, V.; Bermejo Gómez, A.; Ayats, C.; Platero-Prats, A. E.; Carson, F.; Su, J.; Yao, Q.; Pericàs, M. A.; Zou, X.; MartínMatute, B. n., Double-supported silica-metal-organic framework palladium nanocatalyst for the aerobic oxidation of alcohols under batch and continuous flow regimes. ACS Catal. 2014, 5 (2), 472-479, DOI

37. Pascanu, V.; Hansen, P. R.; Bermejo Gomez, A.; Ayats, C.; Platero-Prats, A. E.; Johansson, M. J.; Pericas, M. A.; MartinMatute, B., Highly functionalized biaryls via Suzuki-Miyaura crosscoupling catalyzed by Pd@MOF under batch and continuous flow regimes. ChemSusChem 2015, 8 (1), 123-130, DOI $10.1002 /$ cssc. 201402858 .

38. Yuan, N.; Pascanu, V.; Huang, Z.; Valiente, A.; Heidenreich, N.; Leubner, S.; Inge, A. K.; Gaar, J.; Stock, N.; Persson, I.; Martin-Matute, B.; Zou, X., Probing the Evolution of Palladium Species in Pd@MOF Catalysts during the Heck Coupling Reaction: An Operando X-ray Absorption Spectroscopy Study. J. Am. Chem. Soc. 2018, 140 (26), 8206-8217, DOI 10.1021/jacs.8b03505.

39. Puthiaraj, P.; Ahn, W.-S., Highly active palladium nanoparticles immobilized on $\mathrm{NH}_{2}$-MIL-125 as efficient and recyclable catalysts for Suzuki-Miyaura cross coupling reaction. Catal. Commun. 2015, 65, 91-95, DOI 10.1016/j.catcom.2015.02.017.

40. Shang, N.; Gao, S.; Zhou, X.; Feng, C.; Wang, Z.; Wang, C., Palladium nanoparticles encapsulated inside the pores of a metalorganic framework as a highly active catalyst for carbon-carbon crosscoupling. RSC $A d v$. 2014, 4 (97), 54487-54493, DOI $10.1039 / \mathrm{c} 4 \mathrm{ra} 10065 \mathrm{~d}$

41. Carson, F.; Pascanu, V.; Bermejo Gomez, A.; Zhang, Y.; Platero-Prats, A. E.; Zou, X.; Martin-Matute, B., Influence of the Base on Pd@MIL-101- $\mathrm{NH}_{2}(\mathrm{Cr})$ as Catalyst for the Suzuki-Miyaura CrossCoupling Reaction. Chem. - Eur. J. 2015, 21 (30), 10896-10902, DOI 10.1002/chem. 201500843

42. Dong, W.; Feng, C.; Zhang, L.; Shang, N.; Gao, S.; Wang, C.; Wang, Z., Pd@UiO-66: An Efficient Catalyst for Suzuki-Miyaura Coupling Reaction at Mild Condition. Catal. Lett. 2015, 146 (1), 117125, DOI 10.1007/s10562-015-1659-4

43. Bugaev, A. L.; Guda, A. A.; Lomachenko, K. A.; Kamyshova, E. G.; Soldatov, M. A.; Kaur, G.; Oien-Odegaard, S.; Braglia, L.; Lazzarini, A.; Manzoli, M.; Bordiga, S.; Olsbye, U.; Lillerud, K. P.; Soldatov, A. V.; Lamberti, C., Operando study of palladium nanoparticles inside UiO-67 MOF for catalytic hydrogenation of hydrocarbons. Faraday Discuss. 2018, 208 (0), 287306, DOI $10.1039 / \mathrm{c} 7 \mathrm{fd} 00224 \mathrm{f}$.

44. Huang, Y.; Gao, S.; Liu, T.; Lü, J.; Lin, X.; Li, H.; Cao, R., Palladium Nanoparticles Supported on Mixed-Linker MetalOrganic Frameworks as Highly Active Catalysts for Heck Reactions. ChemPlusChem 2012, 77 (2), 106-112, DOI 10.1002/cplu.201100021.

45. Zhang, L.; Su, Z.; Jiang, F.; Zhou, Y.; Xu, W.; Hong, M., Catalytic palladium nanoparticles supported on nanoscale MOFs: a highly active catalyst for Suzuki-Miyaura cross-coupling reaction. Tetrahedron 2013, $69 \quad$ (44), 9237-9244, $\quad$ DOI 10.1016/j.tet.2013.08.059.

46. Tahmasebi, S.; Mokhtari, J.; Naimi-Jamal, M. R.; Khosravi, A.; Panahi, L., One-step synthesis of Pd$\mathrm{NPs} @ \mathrm{Cu}_{2}(\mathrm{BDC})_{2} \mathrm{DABCO}$ as efficient heterogeneous catalyst for the 
Suzuki-Miyaura cross-coupling reaction. J. Organomet. Chem. 2017, 853, 35-41, DOI 10.1016/j.jorganchem.2017.10.011.

47. Dong, W.; Zhang, L.; Wang, C.; Feng, C.; Shang, N.; Gao, S.; Wang, C., Palladium nanoparticles embedded in metal-organic framework derived porous carbon: synthesis and application for efficient Suzuki-Miyaura coupling reactions. RSC Adv. 2016, 6 (43), 37118-37123, DOI 10.1039/c6ra00378h.

48. Titov, K.; Zeng, Z.; Ryder, M. R.; Chaudhari, A. K.; Civalleri, B.; Kelley, C. S.; Frogley, M. D.; Cinque, G.; Tan, J. C., Probing Dielectric Properties of Metal-Organic Frameworks: MIL53(Al) as a Model System for Theoretical Predictions and Experimental Measurements via Synchrotron Far- and Mid-Infrared Spectroscopy. J. Phys. Chem. Lett. 2017, 8 (20), 5035-5040, DOI 10.1021/acs.jpclett.7b02003.

49. Civalleri, B.; Napoli, F.; Noel, Y.; Roetti, C.; Dovesi, R., Ab-initio prediction of materials properties with CRYSTAL: MOF-5 as a case study. CrystEngComm 2006, 8 (5), 364-371, DOI $10.1039 / \mathrm{b} 603150 \mathrm{c}$

50. Ravel, B.; Newville, M., ATHENA, ARTEMIS, HEPHAESTUS: data analysis for X-ray absorption spectroscopy using IFEFFIT. J. Synchrotron Radiat. 2005, 12 (Pt 4), 537-541, DOI 10.1107/S0909049505012719.

51. Zhao, F.; Bhanage, B. M.; Shirai, M.; Arai, M., Heck Reactions of Iodobenzene and Methyl Acrylate with Conventional Supported Palladium Catalysts in the Presence of Organic and/or Inorganic Bases without Ligands. Chem. - Eur. J. 2000, 6 (5), 843-848, DOI $\quad 10.1002 /($ sici)1521-3765(20000303)6:5<843::aidchem843>3.0.co;2-g.
52. Borja, G.; Monge-Marcet, A.; Pleixats, R.; Parella, T.; Cattoen, X.; Man, M. W. C., Recyclable Hybrid Silica-Based Catalysts Derived from Pd-NHC Complexes for Suzuki, Heck and Sonogashira Reactions. Eur. J. Org. Chem. 2012, (19), 3625-3635, DOI $10.1002 /$ ejoc. 201200205 .

53. Genelot, M.; Dufaud, V.; Djakovitch, L., Carbonylative Sonogashira Coupling in the Synthesis of Ynones: A Study of "Boomerang" Phenomena. Adv. Synth. Catal. 2013, 355 (13), 26042616, DOI 10.1002/adsc. 201300357.

54. Gruttadauria, M.; Giacalone, F.; Noto, R., "Release and catch" catalytic systems. Green Chem. 2013, 15 (10), 2608-2618, DOI $10.1039 / \mathrm{c} 3 \mathrm{gc} 41132 \mathrm{j}$.

55. Giacalone, F.; Campisciano, V.; Calabrese, C.; La Parola, V.; Syrgiannis, Z.; Prato, M.; Gruttadauria, M., Single-Walled Carbon Nanotube-Polyamidoamine Dendrimer Hybrids for Heterogeneous Catalysis. ACS Nano 2016, 10 (4), 4627-36, $\quad$ DOI 10.1021/acsnano.6b00936.

56. Dent, A.; Cibin, G.; Ramos, S.; Smith, A.; Scott, S.; Varandas, L.; Pearson, M.; Krumpa, N.; Jones, C.; Robbins, P. In B18: A core XAS spectroscopy beamline for Diamond, J. Phys.: Conf. Ser., IOP Publishing: 2009; p 012039.

57. Pentsak, E. O.; Kashin, A. S.; Polynski, M. V.; Kvashnina, K. O.; Glatzel, P.; Ananikov, V. P., Spatial imaging of carbon reactivity centers in $\mathrm{Pd} / \mathrm{C}$ catalytic systems. Chem. Sci. 2015, 6 (6), 3302-3313, DOI 10.1039/c5sc00802f. 
Insert Table of Contents artwork here

OX-1 metal-organic framework nanosheets hosting catalytically-active species efficiently catalyse Suzuki coupling and Heck arylation reactions.

(16 words)

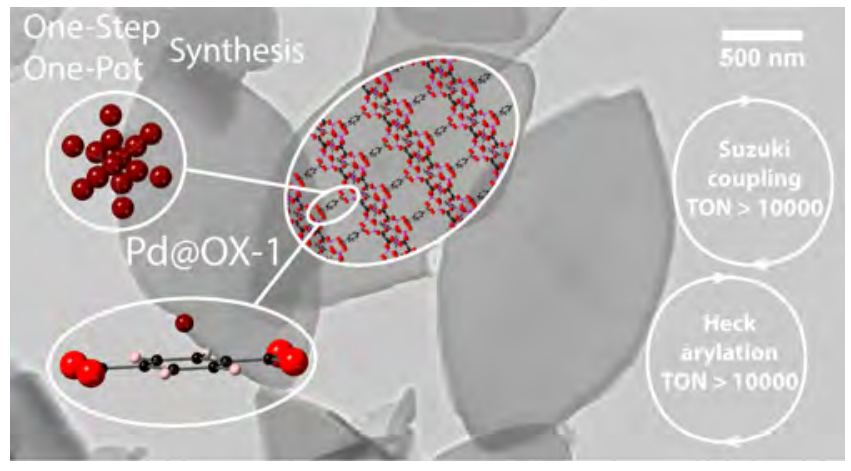

